\title{
Digestibilidad aparente de materia seca, proteína y energía de harina de vísceras de pollo, quinua y harina de pescado en tilapia nilótica, Oreochromis niloticus
}

\section{Apparent digestibility of dry matter, protein and energy regarding fish meal, poultry by-product meal and quinua for Nile tilapia, Oreochromis niloticus}

\author{
Mariana C. Gutiérrez-Espinosa1*, Martha I. Yossa-Perdomo* \\ Walter Vásquez-Torres ${ }^{3 *}$ \\ ${ }^{1}$ Zootecnista, MSc, ${ }^{2}$ Zootecnista, MSc, Dr.Sci, ${ }^{3}$ Biólogo, MSc, Dr.Sci \\ *Grupo de Investigación Granac, Instituto de Acuicultura Universidad de Los Llanos, Colombia \\ Email:marianacgutierreze@gmail.com
}

Recibido: Octubre 25 de 2010. Aprobado: Mayo 4 de 2011

\section{RESUMEN}

El presente estudio se realizó con el objeto de determinar los coeficientes de digestibilidad aparente (CDA) de la proteína (CDAp) y de la energía (CDAe) de harina de pescado (HP), harina de vísceras de pollo (HVP) y harina de quinua Chenopodium quinoa (HQ), para tilapia nilótica Oreochromis niloticus. Para estimar los coeficientes se utilizó el método indirecto con marcador (óxido de cromo), colectando las heces por el sistema Guelph modificado. Tilapias con peso promedio de $130 \mathrm{gr}$ se alimentaron con una dieta experimental constituida por dieta referencia (DR) (89.5\%, $79.5 \%$ ó $69.5 \%), 0.5 \%$ de marcador inerte y el ingrediente a evaluar en un nivel de inclusión de $10 \%, 20 \%$ ó $30 \%$. Las heces comenzaron a colectarse 10 horas después de suministrar el alimento, cada hora durante 12 horas; las muestras se secaron a una temperatura de $60^{\circ} \mathrm{C}$ y se almacenaron a $-17^{\circ} \mathrm{C}$ hasta ser analizadas. Los CDAp para los tres niveles de inclusión (10, 20 y $30 \%$ ) fueron respectivamente: 88.1, 89.4 y $90.9 \%$ para HP; 96.8, 94.9 y 94.1 para HVP y $67.7,73.5$ y 77.5 para HQ. No se observaron diferencias significativas $(p<0.05)$ en los CDAp entre niveles de inclusión en las tres materias primas investigadas. En cuanto a la fracción energética, los resultados para HP se observaron entre $53.0 \%$ y $88.5 \%$, para HVP entre 52.6 y 59.7 y para HQ entre $29.0 \%$ y $66.1 \%$. El $30 \%$ de inclusión de HP presentó el mayor CDAe $(\mathrm{p}<0.05)$. Para HVP no hubo diferencias significativas entre niveles de inclusión; para $\mathrm{HQ}$ el CDAe fue significativamente menor ( $p<$ 0.05) con el nivel de inclusión del $10 \%$.

Palabras claves: Coeficientes de digestibilidad aparente, nutrición, materias alternativas, análisis proximal. 


\section{ABSTRACT}

The purpose of this study was to determine protein (ADCp) and energy (ADCe) apparent digestibility coefficients $(A D C)$ for fish meal (FM), poultry by-product meal (PBM) and quinoa flour (Chenopodium quinoa) (QF) used in feeding Nile tilapia (Oreochromis niloticus). The indirect method with a chromium oxide marker was used to estimate the aforementioned ADC; faeces were collected by the modified Guelph system. Tilapias having an average $130 \mathrm{~g}$ weight were fed on an experimental diet consisting of reference diet (RD) $(89.5 \%, 79.5 \%$ or $69.5 \%), 0.5 \%$ inert marker (chromium dioxide) and the ingredient to be evaluated to assess $10 \%, 20 \%$ or $30 \%$ inclusion level. The fish were fed every hour for 12 hours per day and their faeces were collected 10 hours after the fish had been fed; the samples were dried at $60^{\circ} \mathrm{C}$ and stored at $-17^{\circ} \mathrm{C}$ until analysed. Chromium oxide level was determined by the acid digestion method. The ADCp for the three inclusion levels (10 \% $20 \%$ and $30 \%$ ) were respectively: $88.1 \%, 89.4 \%$ and 90.9 $\%$ for FM, $96.8 \%, 94.9 \%$ and $94.1 \%$ for PBM and $67.7 \%, 73.5 \%$ and $77.5 \%$ for QF. There were no significant differences $(p<0.05)$ concerning ADCp inclusion levels regarding the three materials being investigated. As for ADCe, the results ranged from $53.0 \%$ to $88.5 \%$ for FM, 52.6 \% to $59.7 \%$ for PBM and $29.0 \%$ to $66.1 \%$ for QF. Including $30 \%$ FM had the highest ADCe ( $p>0.05)$; there were no significant differences between PBM inclusion levels and QF was significantly lower (10\% inclusion level).

Key words: Apparent digestibility coefficient, nutrition, alternative matter, proximate analysis.

\section{INTRODUCCIÓN}

La tilapia nilótica, Oreochromis niloticus, tiene un considerable potencial en la acuicultura de las regiones tropicales y subtropicales. Para el año 2004, la FAO, (2006) ubicó a Colombia dentro de los diez principales productores de tilapia del mundo, consolidándose como la principal especie de cultivo con una producción de 27.953 toneladas para ese año. De la producción piscícola total del Departamento del Meta el $63.72 \%$ es tilapia (ICC, 2009).

Dentro de los costos de producción, la alimentación constituye el rubro más importante, excediendo el $70 \%$ de los gastos totales debido a la gran complejidad de los alimentos requeridos en acuicultura, justificando así la necesidad de profundizar en los aspectos de nutrición y alimentación (Abimorad y Carneiro, 2004; Cavalheiro et al. 2007; Pereira-Filho 1995).

Los parámetros para determinar el valor nutricional de los insumos y de las dietas empleadas en la alimentación acuícola son, entre otros, la composición química del ingrediente, las necesidades de la especie y la digestibilidad (Gonçalves y Carneiro, 2003; Manríquez 1994;
Pezzato et al. 2002). La digestibilidad puede variar en función del nivel de inclusión del ingrediente en la dieta y generalmente tiende a disminuir en la medida en que aumenta su concentración en la dieta (Furuichi y Yone, 1980; Gonçalves y Carneiro, 2003; Henken et al. 1985). Otros factores también pueden influir como la especie, edad de los peces, las condiciones fisiológicas, la temperatura del agua, la composición de la dieta, la frecuencia de la alimentación y el origen de la materia prima (Abimorad y Carneiro, 2004; Hepher 1988). La digestibilidad es considerada como uno de los aspectos más importantes en la evaluación eficiente de los ingredientes y requisito para formulación de dietas biológica y económicamente óptimas (Aksnes 1998; Da Silva y Oliva-Teles 1998; Degani et al. 1997; Pezzato et al. 2004; Shipton y Britz, 2001).

En la determinación de exigencias nutricionales, así como en las pruebas para medir digestibilidad, se utilizan como base de las dietas experimentales Dietas Referencia (DR), de preferencia desarrollada para una especie en particular o grupo de peces con hábitos alimenticios similares. Tales DR son elaboradas con ingredientes semipurificados para 
posibilitar mayor control sobre su composición química; para el caso de los experimentos de digestibilidad de materias primas (MP) específicas, la DR es sustituida en un determinado porcentaje por la MP permitiendo de esta manera evaluar su digestibilidad en diferentes niveles de inclusión (Aksnes y Opstvedt, 1998 ; Aksnes et al. 1996; Boscolo et al. 2002; Lanna

\section{MATERIALES Y MÉTODOS}

\section{Localización}

El trabajo se llevó a cabo en el Laboratorio Experimental de Alimentación y Nutrición de Peces (LEANP) de la Estación Piscícola del Instituto de Acuicultura de la Universidad de los Llanos (IALL), ubicado en el kilómetro 4 vía Puerto López, en la vereda Barcelona del Municipio de Villavicencio, Departamento del Meta, Colombia (Latitud $4^{\circ} 4^{\prime} 24.95 " \mathrm{~N}$, Longitud 7334'56.80"O), a 418 m.s.n.m.

\section{Material biológico e infraestructura}

Se utilizaron alevinos machos de Tilapia nilótica (Oreochromis niloticus) variedad chitralada, adquiridos en una granja comercial de la región; estos fueron alimentados con ración comercial (34 \% PB) en la Estación Piscícola del IALL hasta que alcanzaron un peso promedio de $130 \mathrm{~g}$; de los estanques en tierra se seleccionaron tres lotes homogéneos en peso y se distribuyeron en tres piletas circulares de $3500 \mathrm{~L}$ de capacidad, lugar en donde permanecieron por dos semanas como período de adaptación a las condiciones experimentales, alimentados con DR (32 $\%$ de PB). Durante el periodo experimental los tanques cónicos para la recolección de heces fueron abastecidos con agua proveniente de un sistema de recirculación cerrado compuesto por cuatro biofiltros en serie, manteniendo un flujo continuo de $1 \mathrm{l} / \mathrm{min} /$ tanque; la aireación fue permanente en el conjunto de filtros y en los tanques para mantener niveles de oxígeno et al. 2004; Sales y Britz, 2002; Vásquez-Torres et al. 2002).

El objeto de este estudio fue determinar y comparar la digestibilidad aparente de la materia seca, proteína y energía de harina de pescado, harina de vísceras de pollo y quinua, en tres niveles de inclusión en tilapia nilótica.

próximos a saturación (7.9ppm). Con este sistema cerrado se controlaron y mantuvieron estables los parámetros fisicoquímicos del agua (temperatura $25 \pm 1,2$ ${ }^{\circ} \mathrm{C}, \mathrm{pH} 7,1 \pm 0,2$, dureza $>40$ ppm y concentración de nitritos, nitratos y amonio $<0,02 \mathrm{ppm}$ ); semanalmente se registraron dichos parámetros utilizando una sonda Orion 5 Star Thermo Electron Corporation.

\section{Dietas experimentales y alimentación}

Para determinar los CDA de cada una de las MP en estudio, HP, HVP y HQ (Tabla 1), fueron elaboradas raciones constituidas por una mezcla de $\mathrm{DR}$ propuesta por Vásquez-Torres etal. (2002) (Tabla2) y de la MPa evaluar. Se utilizó como marcador inerte el óxido de cromo III $\left(\mathrm{Cr}_{2} \mathrm{O}_{3}\right)$ adicionado a la dieta en una concentración del $0.5 \%$; para cada MP se evaluaron tres diferentes niveles de inclusión 10\%, 20\% y $30 \%$ (Tabla 3). Para la elaboración de estas dietas las MP fueron molidas (partículas 0.2-0.3 mm de diámetro), mezcladas y sometidas a proceso de extrucción a $123^{\circ} \mathrm{C}$ (micro-extrusora Exteec $\AA$, Riberão Preto-Brasil) para formar gránulos compactos, flotantes y con un diámetro de 5-6 $\mathrm{mm}$. Las dietas fueron secadas en horno a $60^{\circ} \mathrm{C}$ por 12 horas y almacenadas en bolsas de cierre hermético a $4^{\circ} \mathrm{C}$ hasta su uso. Acada lote de peces se le suministró una dieta experimental hasta aparente saciedad, dos veces al día (8:00 a.m. y 4:00 p.m.) por un período de 5 días.

Tabla 1. Composición proximal de las materias primas utilizadas

\begin{tabular}{lccccc}
\hline & & \multicolumn{3}{c}{$\mathbf{~ M S}$} & EB \\
\cline { 3 - 5 } \multicolumn{1}{c}{ Materia Prima } & MS (\%) & PB & EE & CZ & (Kcal/Kg) \\
\hline Harina de pescado & 90.9 & 71.73 & 9.5 & 12.4 & 5078.8 \\
Harina de vísceras de pollo & 96.8 & 61.14 & 21.6 & 9.7 & 5535.7 \\
Harina de quinua & 91.7 & 13.08 & 8.3 & 2.7 & 4372.9 \\
\hline
\end{tabular}


Tabla 2. Composición de ingredientes de la dieta referencia (\% en base húmeda)

\begin{tabular}{lr}
\hline \multicolumn{1}{c}{ Ingredientes } & g/100 g dieta \\
\hline Caseína $^{1}$ & 33.3 \\
Gelatina $^{2}$ & 3.4 \\
Dextrina & 40.0 \\
Alfa-Celulosa & 14.1 \\
Aceite pescado & 2.4 \\
Aceite vegetal & 2.4 \\
Premezcla Vitaminas $^{3}$ & 0.2 \\
Premezcla Microminerales $^{4}$ & 0.1 \\
Premezcla Macrominerales $^{5}$ & 4.0 \\
Vitamina C $^{6}$ & 0.1 \\
\hline
\end{tabular}

1 Composición analizada: MS 93 \%; PB 86,42\%; lípidos 2,29 \%, cenizas 3,66 \%

2 Composición analizada: MS $91 \%$; PB 94,02\%.

3 Rovimix Vitaminas Lab. Roche S.A: Vit A 8.0*106 UI, Vit D3, $1.8^{*} 106 \mathrm{UI}$, Vit E $66.66 \mathrm{~g}$, Vit B1 6.66 g, Vit B2 $13.33 \mathrm{~g}$, Vit B6 $6.66 \mathrm{~g}$, Pantotenato de Ca $33.33 \mathrm{~g}$, Biotina $533.3 \mathrm{mg}$, Ac. Fólico $2.66 \mathrm{~g}$, Ac. Ascórbico $400.0 \mathrm{~g}$, Ac. Nicotínico $100.0 \mathrm{~g}$, Vit B12 $20.0 \mathrm{mg}$, Vit K3 $6.66 \mathrm{~g}$, vehículo csp $1.0 \mathrm{~kg}$. 4 Premix microminerales Lab. Roche S.A.: Composición por 100 g: Magnesio 1.0, Zinc 16.0, Hierro 4.0, Cobre 1.0, lodo 0.5, Selenio 0.05. Cobalto 0.01 .

5 Composición por 100 g de mezcla: Ca(H2PO4) 13,6 g; Lactato de Ca 34,85 g; 2MgSO4.7 H2O, 13,2 g; KH2PO4 24.g; NaCl 4.5 g; AlCl3 0.015 g, CMC 9.835 g.

6 Rovimix Stay-C35, DSM.

Tabla 3. Composición proximal de la dieta referencia y las dietas experimentales

\begin{tabular}{lccccc}
\hline \multicolumn{1}{c}{ Dieta } & $\begin{array}{l}\text { MS } \\
\text { (\%) }\end{array}$ & PB & EE & CZ & (Kcal/Kg) \\
& 89.5 & 29.9 & 2.2 & 11.3 & 4118.6 \\
Dieta Referencia & 88.3 & 33.8 & 3.8 & 11.8 & 4398.8 \\
Harina de pescado 10\% & 92.5 & 40.4 & 4.2 & 11.7 & 4385.2 \\
Harina de pescado 20\% & 90.3 & 45.9 & 5.0 & 12.2 & 4517.1 \\
Harina de pescado 30\% & 91.2 & 33.1 & 4.6 & 10.1 & 4427.9 \\
Harina de vísceras de pollo 10\% & 88.3 & 34.6 & 8.1 & 10.4 & 4564.1 \\
Harina de vísceras de pollo 20\% & 90.9 & 39.7 & 9.8 & 10.6 & 4695.9 \\
Harina de vísceras de pollo 30\% & 84.8 & 27.1 & 4.3 & 9.4 & 4346.4 \\
Harina de quinua 10\% & 87.9 & 26.4 & 3.7 & 8.7 & 4227.6 \\
Harina de quinua 20\% & 89.7 & 24.0 & 3.0 & 8.1 & 4227.0 \\
\hline Harina de quinua 30\% & & & & & \\
\hline
\end{tabular}




\section{Recolección de heces}

Al quinto día, después de administrar la ración de la mañana, los peces fueron transferidos a tanques de fondo cónico (Sistema Guelph modificado), colocando 20 animales por tanque de 200 L. Pasadas 10 horas, tiempo que corresponde al tránsito gastrointestinal para esta especie (Lanna et al. 2004), se inició la recolección de las heces en intervalos de una hora, durante 12 horas. Una vez terminada la coleta, el lote de peces fue devuelto a un tanque circular para iniciar, después de un periodo de reposo de 4 días un nuevo experimento con otra dieta experimental. Cada lote de peces fue utilizado tres veces.

\section{Análisis de laboratorio}

Todos los análisis de composición proximal de MP, $\mathrm{DR}$, dietas experimentales y heces, fueron realizados con base en materia seca (MS) y siempre con tres réplicas. Las heces, inmediatamente colectadas se les retiró el exceso de agua y deshidrataron en horno a $60^{\circ} \mathrm{C} / 24$ horas. Todas las muestras secas fueron pulverizadas en micromolino (Scienceware, USA) y almacenadas a $-17^{\circ} \mathrm{C}$ para los análisis de contenido proteico, valor energético y concentración de óxido de cromo. Para determinar la MS, las muestras se colocaron en una estufa (Memmert) a una temperatura de $105^{\circ} \mathrm{C}$ durante 24 horas (AOAC, 1995), tomando el peso antes y después de secar. La proteína presente en MP, DR, dietas experimentales $y$ heces, fue determinada utilizando el método Kjeldalh (metodología patrón descrita por la AOAC, 1995). La energía bruta fue medida en bomba calorimétrica PARR (121EA, USA). La concentración de óxido de cromo $\left(\mathrm{Cr}_{2} \mathrm{O}_{3}\right)$ se determinó por el método de digestión ácida propuesto por Furukawa and Tsukahara, (1966). Para estimar los coeficientes de digestibilidad aparente de las diferentes MP se empleo el método indirecto, propuesto por Cho et al., (1985). El coeficiente de digestibilidad aparente (CDA) de la proteína y de la energía, tanto de la dieta semipurificada como de las dietas experimentales, se calculó utilizando la ecuación de Nose, (1966).

CDANut $(\%)=100-\left(100 x\left(\frac{\% \operatorname{Cr} 203 d}{\% \operatorname{Cr} 203 h} \times \frac{\% \text { Nuth }}{\% \text { Nutd }}\right)\right)$

Donde:

CDA $_{\text {Nut }}(\%)=$ Coeficiente de digestibilidad aparente del Nutriente

$\% \mathrm{Cr}_{2} \mathrm{O}_{3} \boldsymbol{d}=$ Porcentaje de óxido de cromo de la dieta

$\% \mathrm{Cr}_{2} \mathrm{O}_{3} \boldsymbol{h}=$ Porcentaje de óxido de cromo de las heces

$\%$ Nut $\boldsymbol{h}=$ Porcentaje del nutriente en las heces.

$\%$ Nut $\boldsymbol{d}=$ Porcentaje del nutriente en la dieta

La digestibilidad total de cada materia prima se estableció teniendo en cuenta la ecuación descrita por Bureau et al. (1999):

CDAMp $=$ CDANutde $+\left[(\right.$ CDANutde - CDANutds $\left.) * \frac{X * D d s}{Y * D i n g}\right]$

Donde:

$\operatorname{CDA}_{\mathrm{Mp}}(\%)=$ Coeficiente de digestibilidad aparente del nutriente de la Materia Prima.

$\mathbf{C D A}_{\text {Nutde }}=$ Coeficiente de digestibilidad aparente del Nutriente en la dieta experimental.

$\mathbf{C D A}_{\text {Nutds }}=$ Coeficiente de digestibilidad aparente del Nutriente en la dieta semipurificada.

$\mathbf{D}_{d s}=\%$ del nutriente en la dieta semipurificada

$\mathbf{D}_{\text {ing }}=\%$ del nutriente de la materia prima evaluada.

$\mathbf{X}=$ Proporción de la dieta semipurificada (89.5\%,79.5\% o 69.5\%).

$\mathbf{Y}=$ Proporción de la materia prima (10\%, 20\% ० 30\%). 


\section{Diseño experimental y Análisis estadístico}

Se utilizó un diseño experimental completamente al azar en un arreglo factorial $3 \times 3$, tres ingredientes (HP, HVP y HQ) por tres niveles de inclusión (10\%, $20 \%$, y $30 \%$ ) para un total de 9 tratamientos, con tres réplicas cada uno. Los datos fueron expresados como media \pm DE y fueron transformados por el

\section{RESULTADOS}

La composición proximal de las materias primas evaluadas se presenta en la Tabla 1. Aunque estas materias primas fueron de procedencia nacional, presentaron valores de composición proximal similares a los reportados en la literatura (Aslaksen, et al. 2007; Davies y Gouveia, 2006; Gonçalves y Carneiro, 2003; Guo, et al. 2007; Meurer et al. 2003; Meyhuay 1999; Pezzato, et al. 2002; Romo et al. 2006).

En la Tabla 4 se presentan los CDA de la materia seca, proteína y energía para las MP estudiadas. método arco-seno para posteriormente ser sometidos a ANOVA de una vía para detectar diferencias significativas $(P<0,05)$. Las medias de cada tratamiento fueron comparadas por el test de Tukey. Los resultados fueron analizados mediante el software SAS/STAT® versión 9.0 para Windows.

La comparación entre ingredientes no mostró diferencias significativas entre los valores de digestibilidad de materia seca y proteína de HP y HVP determinados ( $p>0.05$ ) pero si con respecto a la $\mathrm{HQ}$, los cuales fueron estadísticamente menores ( $p<0.05)$; para energía, el mayor CDA se observó en la harina de pescado con $30 \%$ de inclusión y el menor, para la HQ con $10 \%$ de inclusión. Solo en estos dos se observaron diferencias significativas en los coeficientes por efecto de los niveles de inclusión de los ingredientes en estudio.

Tabla 4. Coeficientes de digestibilidad aparente de la harina de pescado (HP), harina de vísceras de pollo (HVP) y harina de quinua (HQ) determinados para Oreochromis niloticus. Los valores representan la media de tres repeticiones $\pm \mathrm{DE}$

\begin{tabular}{ccccc}
\hline Materia prima & $\begin{array}{c}\text { Inclusión } \\
(\%)\end{array}$ & Materia Seca & Proteína & Energía \\
\hline & 10 & $75.9 \pm 1.7^{\mathrm{a}}$ & $88.1 \pm 2.1^{\mathrm{a}}$ & $63.9 \pm 8.0^{\mathrm{b} B}$ \\
$\mathrm{HP}$ & 20 & $74.3 \pm 2.7^{\mathrm{a}}$ & $89.4 \pm 0.6^{\mathrm{a}}$ & $53.0 \pm 1.3^{\mathrm{b} \mathrm{B}}$ \\
& 30 & $71.3 \pm 6.2^{\mathrm{a}}$ & $90.9 \pm 2.4^{\mathrm{a}}$ & $88.5 \pm 0.7^{\mathrm{a}}$ \\
& 10 & $72.3 \pm 4.1^{\mathrm{a}}$ & $96.8 \pm 0.6^{\mathrm{a}}$ & $53.8 \pm 3.1^{\mathrm{b}}$ \\
$\mathrm{HVP}$ & 20 & $78.7 \pm 2.8^{\mathrm{a}}$ & $94.9 \pm 1.2^{\mathrm{a}}$ & $52.6 \pm 4.4^{\mathrm{b}}$ \\
& 30 & $74.1 \pm 6.4^{\mathrm{a}}$ & $94.1 \pm 2.1^{\mathrm{a}}$ & $59.7 \pm 1.0^{\mathrm{b}}$ \\
& 10 & $58.8 \pm 5.5^{\mathrm{b}}$ & $67.7 \pm 7.6^{\mathrm{b}}$ & $29.0 \pm 7.4^{\mathrm{c} \mathrm{B}}$ \\
& 20 & $64.4 \pm 4.2^{\mathrm{a}}$ & $73.5 \pm 5.1^{\mathrm{b}}$ & $52.0 \pm 3.2^{\mathrm{b} \mathrm{A}}$ \\
& 30 & $59.9 \pm 4.1^{\mathrm{b}}$ & $77.5 \pm 2.9^{\mathrm{b}}$ & $66.1 \pm 5.6^{\mathrm{b} \mathrm{A}}$ \\
\hline
\end{tabular}

Letras superíndices minúsculas diferentes en la misma columna indican significancia estadística $(p<0.05)$ en la comparación entre todos los ingredientes; letras superíndices mayúsculas diferentes en la misma columna indican diferencias entre niveles de inclusión para cada materia prima $(p<0.05)$. 


\section{DISCUSIÓN}

Los datos de digestibilidad reflejan el porcentaje de alimento absorbido por el animal y son importantes porque al ser empleados en la formulación se obtiene como resultado dietas con alto valor nutricional y bajo impacto ambiental negativo (Lin et al. 2004). El presente estudio mostró que los CDA de la materia seca (MS) y proteína no fueron afectados por el nivel de inclusión $(P>0.05)$ dentro de la misma materia prima. La digestibilidad de la MS para HP y HVP (>71\%) fue alta y superior a la de $H Q$, lo cual indica que la tilapia utiliza eficientemente materias de origen animal, como lo enunciado por Hanley, (1987). Los CDA de MS para HP obtenidos en este estudio fueron similares a los reportados por Hansen etal. (2007) para Gadus morhua (74 \% y $75 \%$ ), Allan et al. (2000) para Bidyanus bidyanus con valores entre $76.8 \%$ y $93.9 \%$, Masagounder et al. (2009) para Lepomis macrochirus y Micropterus salmoides, $77.6 \%$ y $72.7 \%$ respectivamente, siendo estas últimas especies carnívoras. De otro lado fueron menores que los reportados para la misma especie (87.6\%) por Meurer et al. (2003) y para Piaractus brachypomus (90.1\%) por Fernandes et al. (2004). Vásquez-Torres et al. (2010) obtuvieron CDA de $59.4 \%$ en tilapia roja híbrida y Pezzato et al., (2004) de 51,5\% para Tilapia nilótica, valores que son menores que los observados en este estudio. Para la HVP Ios CDA de la MS están en un rango cercano a los valores reportados por Pezzato et al., (2002) para tilapia nilótica (73.9\%), por Masagounder et al., (2009) para Lepomis macrochirus (83.4\%) y Micropterus salmoides (75.9\%) y por Allan et al. (2000) para Bidyanus bidyanus $86.2 \%$. Por el contrario Meurer et al., (2003) obtuvieron para tilapia nilótica, la misma especie del presente trabajo, un CDA de apenas $62.2 \%$. La HQ presentó CDA de MS mayores que los reportados para otros cereales como trigo (36 $52.9 \%$ ), mijo (53.3\%) y sorgo (36.4\%) en Bidyanus bidyanus (Allan et al. 2000).

Con respecto a los $C D A$ de la proteína para $\mathrm{HQ}$ se obtuvieron coeficientes semejantes a los observados en materias primas como el trigo, el arroz y el maíz (Vásquez-Torres et al. 2010) en tilapia roja. Comparando los CDA de la proteína de HP determinados en el presente experimento con los hallados para esta misma especie por Pezzato et al., (2002) y Meurer et al., (2003), fueron semejantes pero un poco menores que los reportados por Masagounder et al., (2009) en Lepomis macrochirus y Micropterus salmoides y por Allan et al., (2000) en Bidyanus bidyanus. Los CDA para proteína de la HQ estuvieron por encima del $67 \%$, esto deja ver la capacidad de la tilapia, al igual que el Piaractus brachypomus, de digerir bien alimentos tanto de origen vegetal como animal (Fernandes et al. 2004). La HQ presentó CDA para proteína menores que los reportados en Bidyanus bidyanus para mijo (86.2 \%) (Allan et al. 2000). Sklan et al. (2004), reporta en tilapia nilótica CDA de proteína para cereales como cebada, maíz sorgo y trigo de $87.4 \%, 75.1 \%, 85.5 \%$, y 79.5 respectivamente, valores similares a los obtenidos en este estudio para quinua. La digestibilidad de la HP en Pseudoplatystoma coruscans (Gonçalves y Carneiro, 2003), Ictalurus punctatus (Wilson y Poe, 1985), Piaractus mesopotamicus (Abimorad y Carneiro, 2004), Oreochromis niloticus (Furuya M. et al. 2001) y Dicentrarchus labrax (Da Silva y Oliva-Teles, 1998) fue determinada entre $84.1 \%$ y $96 \%$ para la fracción proteica y valores entre 72.8 $\%$ y $94.5 \%$ para energía. Cabe resaltar que la calidad de la harina de pescado varía en función del origen, procesamiento y almacenamiento, entre otros. Esto afecta su digestibilidad, por lo que los valores de digestibilidad de esta materia prima, aun en una misma especie, pueden tener grandes fluctuaciones como ha sido reportado en Oncorhynchus mykiss (Aksnes y Opstvedt, 1998 ). En tilapia nilótica Oreochromis niloticus, se han determinado coeficientes de digestibilidad aparente de diversas materias primas de origen animal; se reportan valores de $78.55 \%$ de digestibilidad de la fracción proteica en harina de pescado, de 73.19 $\%$ en harina de carne, de $87.24 \%$ en harina de vísceras de pollo, de $50.69 \%$ en harina de sangre $y$ de $29.12 \%$ en harina de plumas (Pezzato et al. 2002). De acuerdo con Abimorad y Carneiro (2004) y Köprücü y Özdemir (2005), la calidad de la proteína de los ingredientes es el principal factor que afecta su rendimiento y su digestibilidad. 
En relación a la energía hubo diferencias entre los niveles de inclusión para la HP y HQ. Tanto para la HP como la HVP el valor más bajo se presentó en el nivel de inclusión del $20 \%$. Con respecto al $30 \%$ de inclusión el CDA de energía de la HP fue similar al reportado por Sklan et al., (2004) y Meurer et al., (2003) en tilapia nilótica, $89.2 \%$ y $89.5 \%$ respectivamente; pero para la HVP, el valor obtenido fue menor en este estudio (59.7\%) que el reportado por Sklan et al. (2004), (79.2\%). La HQ presentó un CDA bajo para la energía, circunstancia similar a la reportada en cereales por Allan et al., (2000) en Bidyanus bidyanus, una especie de hábito omnívoro; este hecho se atribuye a que son especies con baja capacidad de digerir ingredientes vegetales con alto contenido de carbohidratos complejos.

La alta digestibilidad de MS y proteína de la HVP observada en esta investigación, mayor que la de la $\mathrm{HP}$, hace que este ingrediente se convierta en una materia alternativa en la sustitución de la HP; de igual manera la $\mathrm{HQ}$ presentó una digestibilidad

\section{AGRADECIMIENTOS}

Instituto de Acuicultura de la Universidad de los Llanos - IALL, Instituto de investigación de la Orinoquía Colombiana - IIOC, Proteínas y energéticos de

\section{REFERENCIAS}

Abimorad E, Carneiro DJ. Métodos de colecta de fezes e determinação dos coeficientes de digestibilidade da fração protéica e da energis dos alimentos para o pacu, Piaractus mesopotamicus (Holmberg, 1887). R Bras Zootec. 2004; 33: 11011109.

Aksnes A, Opstvedt J. Content of digestible energy in fish feed ingredients determined by the ingredient-subtitution method. Aquaculture 1998;161: 45-53.

Aksnes A, Hjertnes T, Opstvedt J. Comparison of two assay methods for determination of nutrient and energy digestibility in fish. Aquaculture 1996;140:343-359. mayor del $67 \%$ en cuanto a la proteína, es por lo tanto una materia prima que puede ser incluida en dietas prácticas para tilapia nilótica. Las diferencias que puedan presentarse entre una materia prima en la misma especie también pueden originarse en el uso de diferentes metodologías experimentales (Allan et al. 2000), la periodicidad y tiempo de recolección de heces (Fernandes et al. 2004; Meurer et al. 2003), diferencias en el procesamiento de las MP (Fernandes et al. 2004), nivel de inclusión, DR empleada, la ecuación empleada para el cálculo de los CDA (Bureau y Hua, 2006, Foster, 1999), entre otros.

Los resultados de este estudio mostraron que la HVP por sus altos CDA, principalmente de proteína, es una materia prima que se puede incluir en la elaboración de dietas de tilapia O. niloticus, como principal fuente de proteína de origen animal o como sustituto de la HP. Igualmente la HQ puede ser utilizada como substitutos de otros cereales, con la ventaja de su mayor contenido de proteína.

Colombia S.A. - Proteicol y al Ministerio de Agricultura y Desarrollo Rural por la cofinanciación del proyecto a través del convenio 018-05 IICA/Unillanos.

Allan GL, Parkinson S, Booth MA, Stone DAJ, Rowland SJ, Frances J, Warner-Smith R. Remplacement of fish meal in diets for Australian silver perch, Bidyanus bidyanus:I Digistibility of alternative ingredienst. Aquaculture 2000; 186:293-310.

AOAC, 1995. Association of Official Analytical Chemists. Official Methods of Analysis 16th. AOAC 0066-961X, Arlington, Va.

Aslaksen MA, Kraugerud OF, Penn M, Svihus B, Denstadli V, Jørgensen HY, Hillestad M, Krogdahl Å, Storebakken T. Screening of nutrient digestibilities and intestinal pathologies in Atlantic salmon, Salmo salar, fed diets with legumes, oilseeds, or cereals. Aquaculture 2007;272:541-555. 
Boscolo W, Hayashi C, Meurer F. Digestibilidades Aparente da Energia e Nutrientes da Alimentos Convencionais e Alternativos para a Talápia do Nilo (Oreochromis niloticus, L.). R Bras Zootec. 2002;31:539-545.

Bureau DP, Hua K. Letter to the Editor of Aquaculture. Aquaculture 2006; 252:103-105.

Bureau DP, Harris AM, Cho CY. Apparent digestibility of rendered animal protein ingredients for rainbow trout (Oncorynchus mykiss). Aquaculture 1999;180:345-358.

Cavalheiro O JM, de Souza O E, Singh B P. Utilization of shrimp industry waste in the formulation of tilapia (Oreochromis niloticus Linnaeus) feed. Bioresource Technology 2007; 98 : $602-606$.

Cho CY, Cowey CB, Watanabe T, 1985. Finfish Nutrition in Asia: Methodological approaches to research and development. IDRC, Ottawa, Ont. (Canadá), 154 pp.

Da Silva GJ, Oliva-Teles A. Apparent digestibility coefficients of feedstuffs in seabass (Dicentrarchus labrax) juveniles. . Aquatic Living Resour 1998;11:187-191.

Davies SJ, Gouveia A. Comparison of yttrium and chromic oxides as inert dietary markers for the estimation of apparent digestibility coefficients in mirror carps Cyprinus carpio fed on diets containing soybean-, maize- and fish-derived proteins. Aquaculture Nutrition 2006;12: 451-458.

Degani G, Viola S, Yehuda Y. Apparent digestibility coefficient of protein sources for carp, Cyprinus carpio L. Aquaculture Research 1997;28: 23-28.

FAO. 2006. State of world aquaculture. Production: environments, species, quantities and values. Fishery and Aquaculture Department. Corporate Document Repository. Fisher Technical paper No. 500 Roma 134 pp.
Fernandes JB, Lochmann R, Bocanegra F. Apparent Digestible Energy and Nutrient Digestibility Coefficients of Diet Ingredients for Pacu Piaractus brachypomus. Journal of the Word Aquaculture Society 2004; 35:237-244.

Foster I. A Note on the Method of Calculating Digestibility Coefficients of Nutrients Provided by Single Ingredients to Feeds of Aquatic Animals. Aquaculture Nutrition 1999; 5: 143-145.

Furuichi M, Yone Y.. Effect of dietary levels on the growth and feed efficiency, the chemical composition of liver and dorsal muscle, and the absorptionof dietary protein and dextrin in fishes. Bull of the Jap Soc of Sci Fish. 1980; 46: 225-229.

Furukawa A, Tsukahara H. On the acid digestion for the determination of chromic oxide as an index substance in the study of digestibility of fish feed. Bulletin of Japanese Society of Scientific Fisheries. Bull of the Jap Soc of Sci Fish 1966; 32: 502-506.

Furuya MW, Pezzato LE, Carvalho de Miranda E, Furuya BVR, Barros MM, Lanna TEA. Digestibilidade Aparente da Energia e Nutrientes do Farelo de Canola pela Tilápia do Nilo (Oreochromis niloticus). R Bras Zootec. 2001; 30: 611-616.

Gonçalves GE, Carneiro DJ. Coeficientes de Digestibilidade Aparente da Proteína e Energia de Alguns Ingredientes Utilizados em Dietas para o Pintado (Pseudoplatystoma coruscans). R Bras Zootec. 2003; 32: 779-786.

Guo J, Wang Y, Bureau DP. Inclusion of rendered animal ingredients as fishmeal substitutes in practical diets for cuneate drum, Nibea miichthioides (Chu, Lo et Wu). Aquaculture Nutrition 2007;13: 81-87.

Hanley F. The digestibility of feedstuffs and effects of feeding seelectivity on digestibility determination in tilapia, Oreochromis niloticus. Aquaculture 1987; 66: 163-179. 
Hansen AC, Rosenlund G, Karlsen $\varnothing$, Koppe W, Hemre Gl. Total replacement of fish meal with plant proteins in diets for Atlantic cod (Gadus morhua L.) I - effect on growth and protein retention. Aquaculture 2007; 272: 599-611.

Henken AM, Kleingeld DW, Tijssen PAT. The effect of feeding level on apparent digestibility of dietary dry matter, crude protein and gross energy in the African catfish Clarias gariepinus (BURCHELL, 1822). Aquaculture 1985; 51:1-11.

Hepher B. 1988. Nutrition of pond fishes. Cambridge University Press, Cambridge, Great Britain, 388 pp.

Köprücü K, Özdemir Y. Apparent digestibility of selected feed ingredients for Nile tilapia (Oreochromis niloticus). Aquaculture 2005; 250: 308-316.

ICC. 2009. Pesca y Acuicultura Colombia 2009 " Informe Técnico Regional Litoral Caribe y Pacifico". Corporación Colombiana Internacional, Ministerio de agricultura y Desarrollo Rural. República de Colombia. 70 pp.

Lanna EA, Pezzato LE, Cecon PR, Furuya WM, Bomfim MA. Digestibilidade Aparente e Trânsito Gastrintestinal em Tilápia do Nilo (Oreochromis niloticus), em Função da Fibra Bruta da Dieta. Revista Brasileira de Zootecnia 2004; 33: 21862192.

Lin H, Liu Y, Tian L, Wang J, Zheng W, Huang J, Chen P. Apparent digestibility coefficients of various feed ingredents for grouper Epinephelus coioides. Journal of the Wordld Aquaculture Society 2004; 25: $134-142$.

Manríquez JA. 1994. La digestibilidad como criterio de evaluación de alimentos su aplicación enpeces y en la conservación del medio ambiente. En Control de calidad de insumos y dietas acuáticas. FAO. México. 67-72.

Masagounder K, Firman JD, Hayward RS, Sun S, Brown PB. Apparent digestibilities of common feedstuffs for bluegill Leponis macrochirus and largemouth bass Micropterus salmoides using individual test ingredients. Aquaculture Nutrition 2009; 15: 29-37.

Meurer F, Hayashi C, Boscolo WR. Digestibilidade Aparente de Alguns Alimentos Protéicos pela Tilápia do Nilo (Oreochromis niloticus). Revista Brasileira de Zootecnia 2003; 32: 1809-2003.

Meyhuay M. 1999. Charper XI: Quinua. In Postharvest operations. AGSI-FAO.

Nose T. 1966. Recents advances in the study of fish digestión in Japan. Symposium on feeding trout and salmon culture II, 17.

Pereira-Filho M. 1995. Alternativas para a alimentação de peixes em cativeiro. Criando peixes na Amazônia.Adalberto Luiz Val e Alexandre Honczaryk. Manaus, INPA. 75-82.

Pezzato LE, de Miranda EC, Barros MM, Furuya M, Quintero LG. Digestibilidade aparente da matéria seca e proteína bruta e a energia digestível de alguns alimentos alternativos pela tilápia do Nilo (Oreochromis niloticus). Acta Scientiarum. Animal Sciences 2004; 26: 329-337.

Pezzato LE, Carvalho de Miranda E, Barros MM, Quintero PLG, Furuya MW, Pezzato AC. Digestibilidade Aparente de Ingredientes pela Tilápia do Nilo (Oreochromis niloticus). R. Bras. Zootec. 2002; 31: 1595-1604.

Romo S, Rosero A, Forero C, Ceron E. 2006. Potencial nutricional de harinas de quinua (Chenopodium quinoa $w$ ) variedad piertal en los andes colombianos. Primera parte. Facultad de Ciencias Agropecuarias 4.

Sales J, Britz PJ. Evaluation of the reference diet substitution method for determination of apparent nutrient digestibility coefficients of feed ingredients for South African abalone (Haliotis midae L.) Aquaculture 2002; 207: 113-123.

Shipton TA, Britz PJ. An assessment of the use of chromic oxide as a marker in protein digestibility 
studies with Haliotis midae L. Aquaculture 2001; 203: 69-83.

Sklan D, Prag T, Lupatsch I. Apparent digestibility coefficients of feed ingredients and their prediction in diets for tilapia Oreochromis niloticus Oreochromis aureus (Teleostei, Cichlidae) Aquaculture Research 2004; 35: 358-364.

Vásquez-Torres W, Pereira Filho M, AreasCastellanos JA. Estudos para Composição de una Dieta Referência Semipurifiocada para Avaliaçião de Exigências Nutricionais em Juvenis de
Pirapitinga, Piaractus brachypomus (Cuvier, 1818). R Bras Zootec. 2002; 31, 283-292.

Vásquez-Torres W, Yossa M, Hernández G, Gutiérrez MC. Digestibilidad aparente de ingredientes de uso común en la fabricación de raciones balanceadas para tilapia roja híbrida (Oreochromis sp) Rev Colomb Cienc Pecu 2010; 23: 207-216.

Wilson R, Poe W. Apparent digestible protein and energy coefficients of common feed ingredients for channel catfish. Prog Fish Cult 1985; 47: 154-158. 